\title{
Spatial asymmetries ("pseudoneglect") in free visual exploration-modulation of age and relationship to line bisection
}

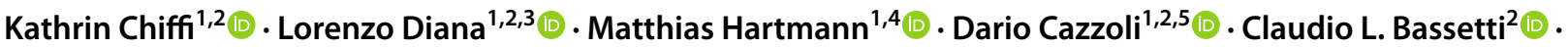 \\ René M. Müri ${ }^{1,2,5} \cdot$ Aleksandra K. Eberhard-Moscicka ${ }^{1,2}$ (1)
}

Received: 6 November 2020 / Accepted: 23 June 2021 / Published online: 4 July 2021

(c) The Author(s) 2021

\begin{abstract}
When humans visually explore an image, they typically tend to start exploring its left side. This phenomenon, so-called pseudoneglect, is well known, but its time-course has only sparsely been studied. Furthermore, it is unclear whether age influences pseudoneglect, and the relationship between visuo-spatial attentional asymmetries in a free visual exploration task and a classical line bisection task has not been established. To address these questions, 60 healthy participants, aged between 22 and 86, were assessed by means of a free visual exploration task with a series of naturalistic, colour photographs of everyday scenes, while their gaze was recorded by means of a contact-free eye-tracking system. Furthermore, a classical line bisection task was administered, and information concerning handedness and subjective alertness during the experiment was obtained. The results revealed a time-sensitive window during visual exploration, between 260 and $960 \mathrm{~ms}$, in which age was a significant predictor of the leftward bias in gaze position, i.e., of pseudoneglect. Moreover, pseudoneglect as assessed by the line bisection task correlated with the average gaze position throughout a time-window of 300-1490 ms during the visual exploration task. These results suggest that age influences visual exploration and pseudoneglect in a time-sensitive fashion, and that the degree of pseudoneglect in the line bisection task correlates with the average gaze position during visual exploration in a time-sensitive manner.
\end{abstract}

Keywords Pseudoneglect · Visual exploration · Eye movements · Line bisection

\section{Introduction}

Communicated by Melvyn A. Goodale.

Aleksandra K. Eberhard-Moscicka

aleksandra.eberhard@neuro.unibe.ch

1 Perception and Eye Movement Laboratory, Departments of Neurology and BioMedical Research, Inselspital, Bern University Hospital and University of Bern, Freiburgstarsse 46, 3010 Bern, Switzerland

2 Department of Neurology, Inselspital, Bern University Hospital, Bern, Switzerland

3 PhD Program in Neuroscience, School of Medicine and Surgery, University of Milano-Bicocca, Monza, Italy

4 Faculty of Psychology, UniDistance Suisse, Brig, Switzerland

5 Gerontechnology and Rehabilitation Group, University of Bern, Bern, Switzerland
Human visual exploration results from a complex interplay between saccadic eye movements and visual fixations. During the exploration of an image, saccades and fixations are typically not homogeneously distributed in space, but are driven by attention, saliency, and other cognitive factors. Healthy subjects may show a leftward bias in the initial phase of the visual exploration of an image (Dickinson and Intraub 2009; Foulsham 2013; Nuthmann and Matthias 2014; Ossandon et al. 2014; Hartmann, 2019). This so-called pseudoneglect has been interpreted as a small, but reliable, asymmetry in the distribution of attention, in which attention is preferentially directed towards the left side (Bowers and Heilman 1980; Jewell and McCourt 2000; Nicholls 2012; Thomas 2015). Pseudoneglect has been mainly reported in line bisection tasks and in visual exploration (Jewell and McCourt 2000; Foulsham et al. 2013; Nuthmann and Matthias 2014). Age may influence performance in such tasks, but the effect of age on 
pseudoneglect is still debated. While some studies reported a reduction or even a directional reversal of pseudoneglect in older healthy adults (Fujii 1995; Failla et al. 2003; Barrett and Craver-Lemley 2008; Schmitz and Peigneux 2011; Benwell 2014), other studies reported no effect of age on this leftward bias; or even a stronger leftward bias with increasing age (De Agostini 1999; Varnava and Halligan 2007; Brooks 2016; Friedrich et al. 2016). Furthermore, other factors such as alertness (Paladini 2017), gender (Friedrich et al. 2018), or handedness (Ossandon et al. 2014) may influence pseudoneglect.

Previous literature indicated that the pseudoneglect arises due to a hemispherical asymmetry between the left hemisphere and the right hemisphere, with visuospatial attention being controlled by frontal-parietal networks. The asymmetry is biased towards the left visual field due to a higher activity of the right hemisphere (Heilman and Van Den Abell 1980; Mesulam 1999; Corbetta and Shulman 2002). Using imaging techniques, such as MRI, it has, furthermore, been shown that for cognitive domains the lateralization of the brain activity is reduced with age and in general more bilateral (Dolcos et al. 2002; Brooks et al. 2016; Ng 2016). This can thus also lead to a reduction of the asymmetry with respect to visual attention between the hemispheres leading to changes of the pseudoneglect.

Further studies, however, have also shown that whether pseudoneglect can reliably be measured is also dependent on the stimulus duration. Studies showing the stimuli only for a small duration (such as $150 \mathrm{~ms}$ or $1000 \mathrm{~ms}$ ) have been able to reliably measure a pseudoneglect with a change in age, while in studies in which no temporal restriction have been given have not always been able to do so (Schmitz and Peigneux 2011; Benwell et al. 2014; Brooks et al. 2016).

By now, landmark task and line bisection were systematically investigated with respect to effects of aging. We add a more naturalistic free visual exploration task that resembles more closely everyday exploration and investigate whether age modulates the asymmetries in free visual exploration.

The goals of the present study were threefold: (a) to assess the temporal dynamics of pseudoneglect during visual exploration of naturalistic everyday scenes; (b) to investigate the influence of age on pseudoneglect; and (c) to determine whether pseudoneglect, as assessed by a classical paper-pencil task, would correlate with pseudoneglect observed in a free visual exploration task. To this end, we tested 60 healthy participants, ranging from young adults to elderly (i.e., 22-86 years of age). Furthermore, we were interested in whether other factors such as gender, handedness, and subjective alertness would modulate visual exploration patterns.

\section{Methods}

\section{Participants}

Sixty neurologically healthy adults participated in this study (age range 22-86 years, 31 women, see Table 1). Participants gave their written informed consent prior to participation. The study was carried out in accordance with the code of ethics of the World Medical Association (Declaration of Helsinki). All participants had normal or corrected-tonormal visual acuity, and participants with a history of eye diseases were excluded from the study. None of the subjects reported any difficulties to clearly perceive the visual stimuli while performing the experimental tasks.

\section{Stimuli and materials}

\section{Free visual exploration task}

In the free visual exploration task, participants viewed a series of naturalistic, coloured photographs of everyday scenes $(N=120)$ in a dimly lit room, while their gaze was recorded by means of a contact-free eye-tracking system (see section Eye tracking below for further details). The images were selected from a free image database (pixabay.com), from the sub-categories "nature" and "architecture". The selection of the images was based on their saliency maps, as assessed by a dedicated algorithm (Itti et al. 1998; Paladini et al. 2017). This algorithm takes into account different characteristics of the features within an image, such as orientation, colour, and intensity, which allow the computation of a map of salient regions within the image. This procedure allowed to balance the overall saliency between the left $(M=31.6, \mathrm{SD}=7.37)$ and the right $(M=32.3, \mathrm{SD}=8.49)$ halves of the images $(t(119)=-0.945, p=0.347)$. Please note that the raw values produced by the algorithm were multiplied by 100 to increase the readability of the results. Moreover, images containing humans or letterings were not included. Two examples of presented photographs are shown in Fig. 1 and the exhaustive choice of the experimental stimuli as well as the ratings produced by the saliency

Table 1 Overview of demographic data of the 60 participants included in the study

\begin{tabular}{llr}
\hline & Mean \pm SD & Range \\
\hline Age (years) & $43.05 \pm 19.60$ & $22-86$ \\
Education (years) & $17.19 \pm 2.99$ & $8-22$ \\
Subjective alertness & $7.36 \pm 1.84$ & $2-10$ \\
Handedness & $88.3 \%(N=53)$ right-handed & \\
Gender & $51.7 \%(N=31)$ females & \\
\hline
\end{tabular}


Fig. 1 Example of stimuli used in the free visual exploration task
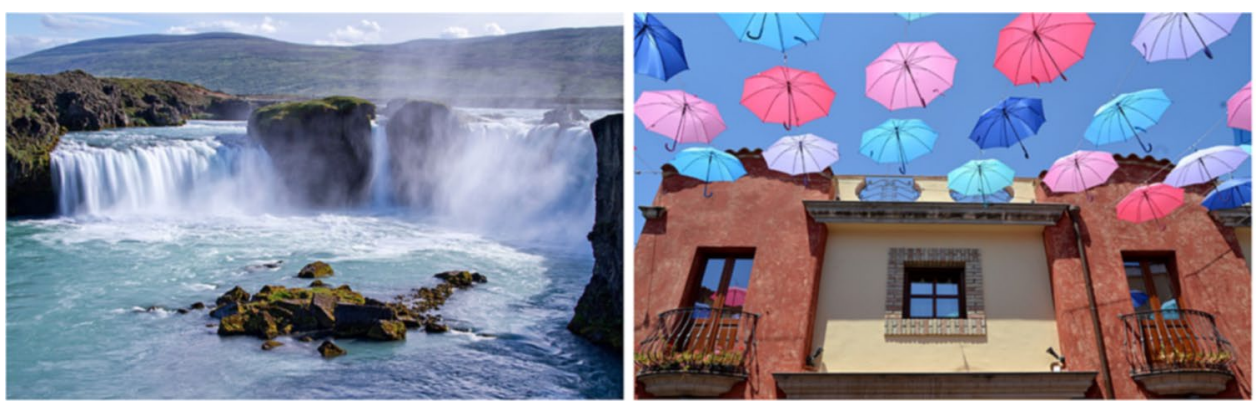

algorithm (Itti et al. 1998) are available at the URL: https:// osf.io/zd3qm/. To avoid fatigue and to allow for periodical calibration of the eye-tracking system, the photographs were distributed into six sets of 20 photographs each. Following a nine-point calibration, the free visual exploration task proceeded by displaying the series of images, one at a time, in a random order. After each set (20 images), participants were allowed to take a short break and the calibration was repeated. Each trial began with a central fixation marker $(1.5 \mathrm{~s})$, followed by an image displayed for $7 \mathrm{~s}$. Participants were instructed to freely explore the images, as if they were looking at photographs in a photo album

\section{Line bisection task}

In a classical line bisection task, participants were presented with twenty horizontal lines of different lengths, printed on a landscape A4 paper sheet; the actual centre of each line varying along the horizontal dimension (Schenkenberg et al. 1980). The centre of the paper sheet was aligned with the participant's midsagittal plane, and participants were instructed to bisect all lines as quickly as possible using their dominant hand. The uppermost and the lowermost lines were used as practice trials. For the remaining 18 lines, the deviation of the bisection mark from the actual centre of the line was measured in $\mathrm{cm}$. This value was further divided by the actual midline (in $\mathrm{cm}$ ) of the respective lines, and then multiplied by 100 , thus yielding a percent deviation; thereby, negative values indicated a left-sided deviation, and positive values a right-sided deviation.

\section{Subjective alertness assessment}

The subjective level of alertness was assessed by means of a visual analogue scale (VAS). On a $10 \mathrm{~cm}$ long vertical line, ranging from "very alert" to "not at all alert", participants were instructed to draw a horizontal mark to indicate how alert they felt. The distance between the lower extreme of the vertical line and the participants' mark was measured in $\mathrm{mm}$, with lower values indicating a subjective lower level of alertness.

\section{Handedness}

Handedness was assessed by the Edinburgh Handedness Inventory (EHI; Oldfield 1971), measuring hand preference by asking participants to choose which hand(s) is used for a range of 10 everyday tasks. The EHI scores range from -100 to 100 , with negative scores indicating a tendency to left-handedness, and a positive score indicating a tendency to right-handedness.

\section{Eye tracking}

In the visual exploration task, participants viewed a series of images that were presented full-screen on a 22 " computer display (Dell, Dell Inc.), with a refresh rate of $60 \mathrm{~Hz}$, a colour-depth of 32 bit, a resolution of $1680 \times 1050$ pixels, and subtending a visual angle of approximately $37.48^{\circ} \times 23.80^{\circ}$. The screen was placed at the eye level, in line with the participants' midsagittal plane and participants were seated approx. $65 \mathrm{~cm}$ from the screen. A contact-free eye-tracking system, equipped with automatic head-movement compensation, was used to record eye movement data (RED 250, SensoMotoric Instruments $\mathrm{GmbH}$ ). The eye position was sampled at $250 \mathrm{~Hz}$, with a spatial resolution of $0.03^{\circ}$ and an average gaze accuracy of $0.4^{\circ}$. Stimulus presentation was controlled by the Experiment Center software (SensoMotoric Instruments $\mathrm{GmbH}$ ), and the iView $\mathrm{X}$ software (SensoMotoric Instruments $\mathrm{GmbH}$ ) was used for eye movement data acquisition. Raw data were parsed into fixations and saccades using the default parameters of the manufacturer's analysis software (BeGaze ${ }^{\mathrm{TM}}$, SensoMotoric Instruments $\mathrm{GmbH}$ ). The results were exported in an open format (.txt) and were analyzed using R (Version 3.5.0) and Matlab 2019b (The MathWorks Inc., Natick 2019).

\section{Data analysis}

To ensure that all scan paths would indeed start from the middle of the images, as enforced by the central fixation cross presented before each image, an offline drift correction was performed. For this purpose, a pixel band of 184 pixels, corresponding to $2^{\circ}$ visual angle, around the vertical 
midline of the image was defined. Images in which the initial fixations started outside of this pixel band were excluded from further analysis (i.e., 472 out of 7159 images). For the remaining fixations, an offline drift correction was applied. To this end, the horizontal deviation from the midline on the $x$ axis was calculated for the last fixation taking place on the fixation cross. Afterwards, all the $x$ values of the fixations of the following trial were shifted by this offset. At last the mean number of fixations as well as the mean fixation duration, were calculated.

To analyse the time course of attentional asymmetries (Nuthmann and Matthias 2014), the average gaze position deviation was computed over $10 \mathrm{~ms}$ bins, i.e., $N=700$ for 7 s (Hartmann et al. 2019). In brief, the horizontal deviation from the centre of the image, i.e., the difference between $\times$ coordinates of the corresponding fixations and the midline, was calculated for every fixation falling within a given $10 \mathrm{~ms}$ bin; the values were then averaged within the corresponding bin. This served as a measurement of attentional asymmetries (Hartmann et al. 2019). Thus, negative values indicate a leftward bias, and positive values a rightward bias. Deviation values were computed for each participant and every time bin. The nonparametric random permutation procedure proposed by Maris and Oostenveld (2007) was implemented to account for the problem of multiple comparisons. With this approach, time bins during which the gaze position could be predicted by the age or the performance in the line bisection task, were defined and tested for significance. Specifically, it was tested for each $10 \mathrm{~ms}$ bin whether age or the performance in line bisection was a significant predictor for the horizontal gaze position. Adjacent $10 \mathrm{~ms}$ bins for which a significant predictor $(p<0.05)$ was found formed a cluster, and Fisher's $F$ values of all bins within a cluster were summed up, resulting in "cluster mass values". These values were then compared to a "random distribution" of mass values that was obtained by computing the highest "by chance significant" cluster mass value from randomly permutated bins for 5000 times. The $p$ value of each initial cluster was then obtained from the position of the cluster mass value within this "random distribution" (see Hartmann et al. 2019). In a next step, the horizontal gaze position was averaged for the time period of significant clusters and correlated with other variables of interest (Spearman's correlations are reported). The permutations, as well as the corresponding $p$ values, were obtained using the R-package "permuco" (Frossard and Renaud 2018).

Moreover, to test whether additional factors such as gender or subjective alertness would influence the visual exploration behaviour, a linear mixed model with factors age, performance in the line bisection, handedness, gender and subjective alertness was calculated. For this analysis, the average gaze position over the whole presentation time was considered, irrespective of its time-course.

\section{Results}

\section{Free visual exploration pattern}

Participants produced on average 21.41 fixations per image $(M=21.41, \mathrm{SD}=4.37)$, with an average gaze fixation duration of $250 \mathrm{~ms}(M=250.41 \mathrm{SD}=43.65)$. Overall, during the initial stages of the exploration, there was a tendency to deviate towards the left side of the image. This initial leftward bias lasted for about $1.5 \mathrm{~s}$, after which the exploration pattern shifted towards the right side of the image. It is worth to note that the maximal deviation from the midline was more pronounced for the left than for the right part of the images, even though overall, participants spent more time on the right than on the left side of the images (Fig. 2).

\section{The effect of age on spatio-temporal asymmetries in the free visual exploration task}

The nonparametric random permutations indicated a significant time cluster at 260-960 ms (cluster mass $=632.6$,

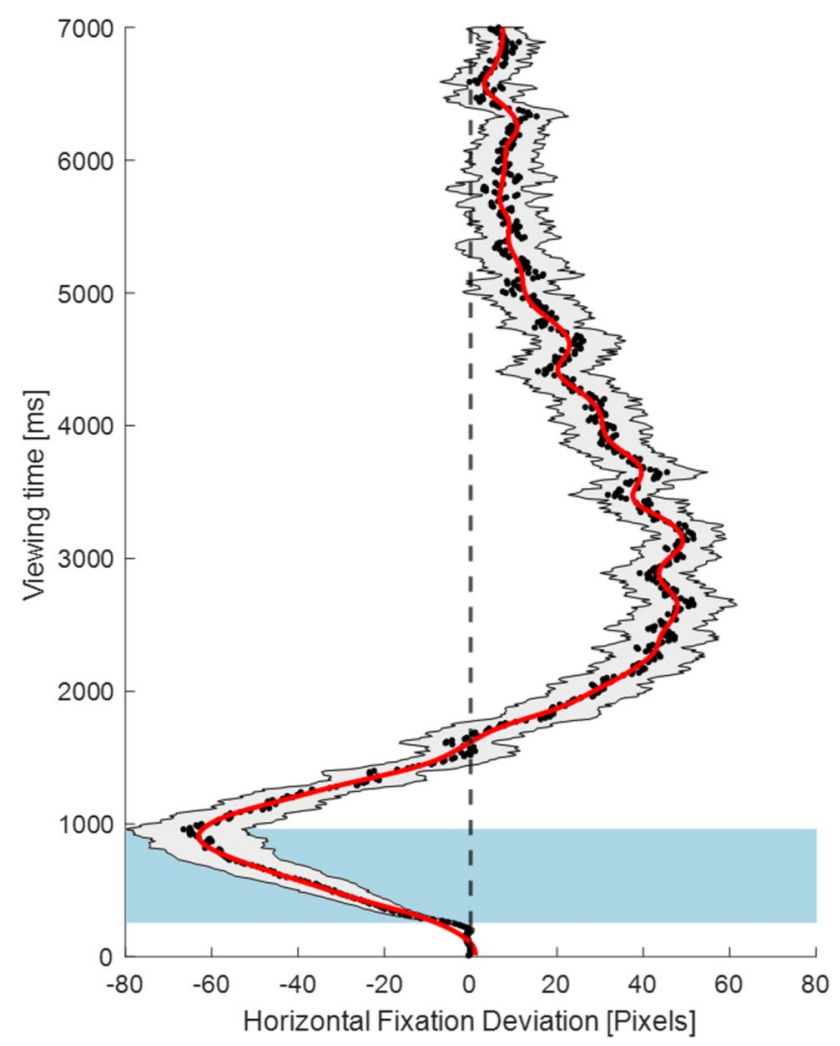

Fig. 2 Time-course of the exploration pattern, averaged across all images and all participants. The grey area around the smoothed red line represents the standard error of the mean of the averaged gaze position. The blue box represents the time-window during which age was a significant predictor for the average gaze position 


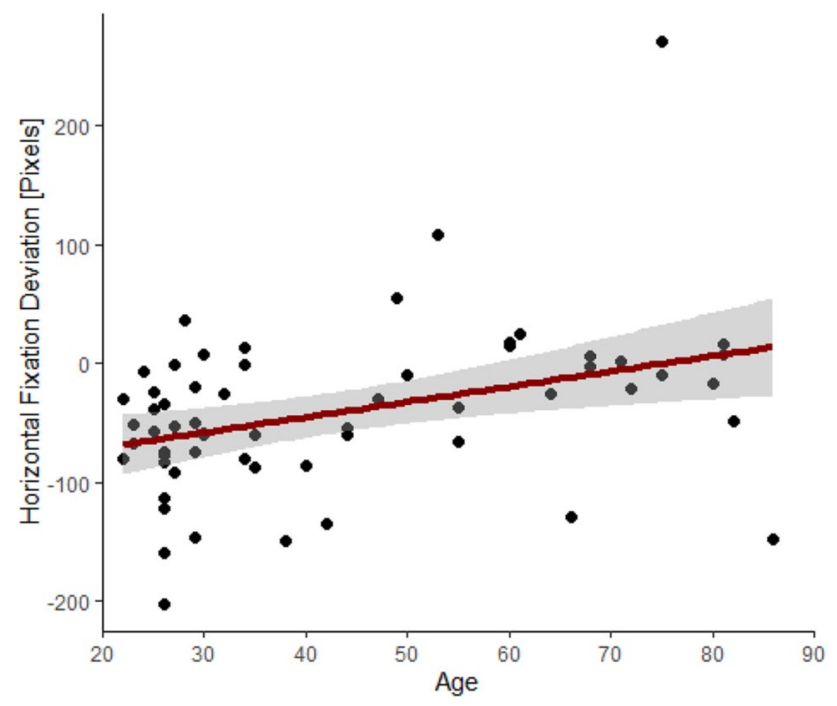

Fig. 3 Correlation between age and the average horizontal gaze deviation in pixels between 260 and $960 \mathrm{~ms}$, indicating attenuation of the leftward bias with increasing age

$p=0.0272$, see Fig. 2). During this early phase of visual exploration, age modulated the exploration behaviour in a way that, with increasing age, the initial leftward bias was attenuated $\left(r_{S}(60)=0.38, p=0.003\right.$, see also Fig. 3$)$.

\section{Visuo-spatial asymmetries in the line bisection task}

We further investigated whether spatial asymmetries in a more naturalistic free visual exploration task would correlate with the performance in a classical test of visuo-spatial attentional bias, namely, the line bisection task.

Overall, the performance in the line bisection task indicated a small leftward bias (relative deviation from the middle: $M=-0.27 \%$; $95 \%$ CI $[-0.97,0.43]$; $S D=2.69 \%$, range $-6.33-5.71 \%)$. The nonparametric random permutations indicated a significant time interval between 300 and $1490 \mathrm{~ms}$ (cluster mass $=795.87, p=0.0218$ ), in which the performance in the line bisection task was positively correlated with the average gaze position $\left(r_{S}(60)=0.27, p=0.034\right.$, see Fig. 4). As such, performance in the line bisection task was predictive of the mean gaze position in the visual exploration task during the initial phase of exploration.

Furthermore, there was no significant correlation between age and the performance in the line bisection task $(r(60)=0.19, p=0.136)$.

\section{Influence of other individual factors on the performance in the free visual exploration task}

A linear mixed model was calculated to test whether, in addition to age and performance in the line bisection task, other

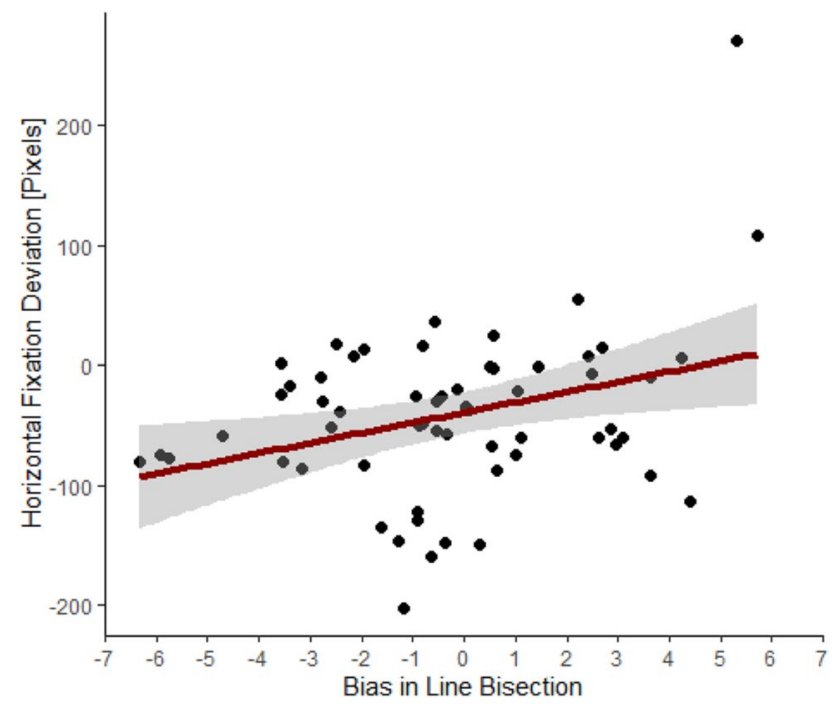

Fig. 4 Correlation between the bias in the line bisection task (percentage deviation from the actual midline) and the average horizontal fixation deviation in the free visual exploration task during the time interval between 300 and $1490 \mathrm{~ms}$; this significant correlation indicates that a stronger leftward bias in the line bisection task correlated with a stronger leftward deviation in the free visual exploration

factors such as gender, handedness, and subjective alertness would influence the exploration behaviour, as measured by the average gaze position. In line with previous analyses, line bisection performance $(p=0.028)$ and age $(p=0.035)$ modulated the average gaze position in the visual exploration task. Yet, none of the additional factors had a significant influence ( $p=0.92$ for gender, $p=0.38$ for handedness, and $p=0.62$ for subjective alertness).

\section{Discussion}

The aim of the present study was to investigate the spatial and temporal dynamics of free visual exploration of complex naturalistic images. Furthermore, we were interested in assessing whether different individual factors such as age, gender, handedness, and subjective alertness would modulate these spatio-temporal dynamics. In agreement with previous studies (Nuthmann and Matthias 2014; Hartmann et al. 2019), we found a pseudoneglect, as manifested by a leftward bias during the initial phase of the free visual exploration of an image. Second, and more importantly, we found a significant influence of age during a critical time window in the early phase of exploration (between 260 and $960 \mathrm{~ms}$ ), i.e., this leftward bias was attenuated with increasing age. Thirdly, we found a significant correlation between the line bisection bias and the spatial bias in free visual exploration, indicating that a stronger leftward bias in the line bisection task correlated with a stronger leftward 
deviation in the visual exploration task. Finally, we found no significant effect of subjective alertness, handedness, or gender on spatio-temporal fixation dynamics during free visual exploration.

To the best of our knowledge, a critical time window during which age-dependent differences in spatial biases are evident in free visual exploration has never been described before in the literature. A leftward bias in the visual exploration behavior is reminiscent of the phenomenon of pseudoneglect. The so-called pseudoneglect is a slight leftward attentional bias, commonly observed, e.g., in the line bisection task. Several studies yielded evidence for an age-related reduction of pseudoneglect, i.e., from a strong attentional leftward bias in young adults to a suppressed or even reversed bias in the elderly, as in our study (see Schmitz and Peigneux 2011 for a review). However, the literature is not conclusive, i.e., several other studies failed to show such age-related changes in spatial biases (Beste et al. 2010; Hatin et al. 2012; Brooks et al. 2016). The results of our study suggest that time is a critical factor, i.e., an age-dependent modulation of spatial biases is only evident in a critical time window; this might explain, at least in part, the discrepancies in the earlier literature.

The null results between age and the line bisection underlie the fact that assessing leftward biases within specific time windows, in line with a characterization of dynamic behaviour with a high temporal resolution, is necessary.

Only few studies examined the influence of age on visual exploration behaviour. Urwyler and colleagues (Urwyler 2015) analysed the influence of age on visual exploration during driving. They found an effect of age, showing that older participants had a narrowed visual exploration field. Furthermore, detection of targets in a visual search task decreased with age, especially for more peripheral targets (Gruber 2014). However, to the best of our knowledge, our study is the first to use a free visual exploration paradigm in participants of different ages.

The origin of the age-related modulation of spatial biases is still debated. It has been suggested that healthy aging might be associated with a functional decline of the right hemisphere, coupled with a left-hemispheric compensation (Dolcos et al. 2002; Schmitz and Peigneux 2011). Indeed, an age-related reduction of the right-hemispheric lateralization has been shown in an EEG study applying a landmark task (Learmonth 2017). Such a relative hyperactivity of the left hemisphere would explain the rightward bias shift in older individuals. This phenomenon can be considered as a less pronounced form of the biased spatial dynamics that have been described in classical neglect models (e.g., Kortman and Nicholls 2016; Delazer 2018). An alternative explanation may be a decline in corpus callosum function, which could impair interhemispheric connectivity. This could then reduce the inhibitory influence that the right hemisphere exhibits in elderly, which would then result in a stronger involvement of the left hemisphere (Schmitz and Peigneux 2011). It has also been proposed that, in elderly individuals yielding comparable behavioural performances as younger adults, the age-related neuronal decline is counteracted by means of plastic reorganization mechanisms (Cabeza 2002). These plastic reorganization mechanisms seem not to take place in all (or at least not to the same extent) elderly individuals. This could, in turn, explain the age-related increase in variability of the free visual exploration pattern our study.

A possible explanation for the critical time window identified by our study, in which age-dependent differences in the visual exploration task were evident, stems from electrophysiological studies. Störmer et al. (Störmer 2013) investigated neural correlates of age-related differences in spatial attention using event-related potentials (ERPs). They found that healthy aging affects attentional selection (supporting the resolution of competition between visual information) at early stages of attentional modulation. To this end, they showed that older adults showed less pronounced selective attentional modulation in the early phase of the visual P1 component (100-125 ms) than younger adults. However, with a $25 \mathrm{~ms}$ delay relative to younger adults, older adults showed distinct processing of targets (125-150 ms), i.e., a delayed yet intact attentional modulation. Moreover, the magnitude of the delayed attentional modulation was related to the behavioural performance in older adults. Further ERP studies on attention in young (Foxe et al. 2003; Longo 2015) and older adults (Learmonth et al. 2017) also indicated a critical time-window, starting as early as $139 \mathrm{~ms}$ after the stimulus presentation. This effect was observed until $400 \mathrm{~ms}$ after the stimulus onset. In addition, an age-dependent time window, ranging from 280 to $400 \mathrm{~ms}$, has been reported (Learmonth et al. 2017), which also coincides with the start of the critical time-window in the present study.

In our study, other factors such as subjective alertness, handedness, or gender did not significantly influence the observed spatial bias. Contrary to the previously mentioned studies, subjects in our study were not specifically recruited to increase variability in the aforementioned factors, which led to a balanced cohort, with only a limited variance with regards to subjective alertness as well as handedness.

In conclusion, our study revealed that, during visual exploration of naturalistic everyday scenes, there is a critical time window within the first second of visual exploration in which age is a predictor of the attenuation of this leftward bias. Furthermore, a significant correlation between line bisection bias and spatial bias during visual exploration was found, i.e., the stronger the leftward bias in line bisection, the stronger the leftward deviation during visual exploration. Hence, our study concurs with previous research by providing evidence that free visual exploration of naturalistic scenes generally starts within the left side of an image, but 
it extends it in two important ways. First, by providing a systematic and detailed time-course investigation of spatial asymmetries during naturalistic scene perception; second, by directly comparing the outcome of two attentional tasks of visuo-spatial nature (i.e., free visual exploration and line bisection task) in a sample of neurologically healthy subjects ranging from young adults to elderly.

Acknowledgements This work was supported by the Swiss National Science Foundation (Grant ID: 160803).

Author contributions All authors contributed to the study conception and design. Material preparation, data collection and analysis were performed by KC, LD, MH and AKE-M. The first draft of the manuscript was written by $\mathrm{KC}$ and all authors commented on previous versions of the manuscript. All authors read and approved the final manuscript.

Funding Open Access funding provided by Universität Bern.

Data availability The data used for the analyses, as well as experimental stimuli used in the visual exploration task and their saliency maps computed by a dedicated algorithm (Itti et al. 1998) are available at the URL: https://osf.io/zd3qm/.

Code availability The code will be made available upon request.

\section{Declarations}

\section{Conflict of interest None.}

Ethical approval This study was conducted with human participants and was given ethical approval by the cantonal ethics committee of the Canton of Bern.

Consent to participate Informed consent was obtained according to the Declaration of Helsinki.

Open Access This article is licensed under a Creative Commons Attribution 4.0 International License, which permits use, sharing, adaptation, distribution and reproduction in any medium or format, as long as you give appropriate credit to the original author(s) and the source, provide a link to the Creative Commons licence, and indicate if changes were made. The images or other third party material in this article are included in the article's Creative Commons licence, unless indicated otherwise in a credit line to the material. If material is not included in the article's Creative Commons licence and your intended use is not permitted by statutory regulation or exceeds the permitted use, you will need to obtain permission directly from the copyright holder. To view a copy of this licence, visit http://creativecommons.org/licenses/by/4.0/.

\section{References}

Barrett AM, Craver-Lemley CE (2008) Is it what you see, or how you say it? Spatial bias in young and aged subjects. J Int Neuropsychol Soc 14(4):562-570. https://doi.org/10.1017/S1355617708080764

Benwell CSY et al (2014) A rightward shift in the visuospatial attention vector with healthy aging. Front Aging Neurosci. https://doi.org/ 10.3389/fnagi.2014.00113
Beste C, Hamm JP, Hausmann M (2010) Developmental changes in visual line bisection in women throughout adulthood. Dev Neuropsychol. https://doi.org/10.1207/s15326942dn3002_6

Bowers D, Heilman KM (1980) Pseudoneglect: effects of hemispace on a tactile line bisection task. Neuropsychologia 18(4-5):491-498. https://doi.org/10.1016/0028-3932(80)90151-7

Brooks JL et al (2016) Adult developmental trajectories of pseudoneglect in the tactile, visual and auditory modalities and the influence of starting position and stimulus length. Brain Cogn 103:12-22. https://doi.org/10.1016/j.bandc.2015.12.001

Cabeza R et al (2002) Aging gracefully: compensatory brain activity in high-performing older adults. Neuroimage 17(3):1394-1402. https://doi.org/10.1006/nimg.2002.1280

Corbetta M, Shulman GL (2002) Control of goal-directed and stimulusdriven attention in the brain. Nat Rev Neurosci 3(3):201-215. https://doi.org/10.1038/nrn755

De Agostini M et al (1999) Comparing left and right hand in line bisection at different ages. Dev Neuropsychol 15(3):379-394. https:// doi.org/10.1080/87565649909540756

Delazer M et al (2018) Eye-tracking provides a sensitive measure of exploration deficits after acute right MCA stroke. Front Neurol. https://doi.org/10.3389/fneur.2018.00359

Dickinson CA, Intraub H (2009) Spatial asymmetries in viewing and remembering scenes: consequences of an attentional bias? Atten Percept Psychophys 71(6):1251-1262. https://doi.org/10.3758/ APP.71.6.1251

Dolcos F, Rice HJ, Cabeza R (2002) Hemispheric asymmetry and aging: right hemisphere decline or asymmetry reduction. Neurosci Biobehav Rev 26(7):819-825. https://doi.org/10.1016/S01497634(02)00068-4

Failla CV, Sheppard DM, Bradshaw JL (2003) Age and respondinghand related changes in performance of neurologically normal subjects on the line-bisection and chimeric-faces tasks. Brain Cogn 52(3):353-363. https://doi.org/10.1016/S0278-2626(03) 00181-7

Foulsham $\mathrm{T}$ et al (2013) Leftward biases in picture scanning and line bisection: a gaze-contingent window study. Vision Res 78:14-25. https://doi.org/10.1016/j.visres.2012.12.001

Foxe JJ, McCourt ME, Javitt DC (2003) Right hemisphere control of visuospatial attention: line-bisection judgments evaluated with high-density electrical mapping and source analysis. Neuroimage 19(3):710-726. https://doi.org/10.1016/S1053-8119(03)00057-0

Friedrich TE, Hunter PV, Elias LJ (2016) Developmental trajectory of pseudoneglect in adults using the greyscales task. Dev Psychol 52(11):1937-1943. https://doi.org/10.1037/dev0000202

Friedrich TE, Hunter PV, Elias LJ (2018) The trajectory of pseudoneglect in adults: a systematic review. Neuropsychol Rev. https:// doi.org/10.1007/s11065-018-9392-6

Fujii T et al (1995) Effect of age on the line bisection test. J Clin Exp Neuropsychol 17(6):941-944. https://doi.org/10.1080/01688 639508402443

Gruber N et al (2014) Effects of age and eccentricity on visual target detection. Front Aging Neurosc. https://doi.org/10.3389/fnagi. 2013.00101

Hartmann M et al (2019) Further to the right: viewing distance modulates attentional asymmetries ("pseudoneglect") during visual exploration. Brain Cogn 129:40-48. https://doi.org/10.1016/j. bandc.2018.11.008

Hatin B, Sykes Tottenham L, Oriet C (2012) The relationship between collisions and pseudoneglect: is it right? Cortex 48(8):997-1008. https://doi.org/10.1016/j.cortex.2011.05.015

Heilman KM, Van Den Abell T (1980) Right hemisphere dominance for attention: the mechanism underlying hemispheric asymmetries of inattention (neglect). Neurology 30(3):327-330. https://doi.org/ $10.1212 / \mathrm{wnl} .30 .3 .327$ 
Itti L, Koch C, Niebur E (1998) A model of saliency-based visual attention for rapid scene analysis. IEEE Trans Pattern Anal Mach Intell 20(11):1254-1259. https://doi.org/10.1109/34.730558

Jewell G, McCourt ME (2000) Pseudoneglect: a review and metaanalysis of performance factors in line bisection tasks. Neuropsychologia 38(1):93-110. https://doi.org/10.1016/S0028-3932(99) 00045-7

Kortman B, Nicholls K (2016) Assessing for unilateral spatial neglect using eye-tracking glasses: a feasibility study. Occup Ther Health Care 30(4):344-355. https://doi.org/10.1080/07380577.2016. 1208858

Learmonth G et al (2017) Age-related reduction of hemispheric lateralisation for spatial attention: an EEG study. Neuroimage 153:139-151. https://doi.org/10.1016/j.neuroimage.2017.03.050

Longo MR et al (2015) Right hemisphere control of visuospatial attention in near space. Neuropsychologia 70:50-357. https://doi.org/ 10.1016/j.neuropsychologia.2014.10.035

Maris E, Oostenveld R (2007) Nonparametric statistical testing of EEG- and MEG-data. J Neurosci Methods 164(1):177-190. https://doi.org/10.1016/j.jneumeth.2007.03.024

Mesulam MM (1999) Spatial attention and neglect: parietal, frontal and cingulate contributions to the mental representation and attentional targeting of salient extrapersonal events. Philos Trans R Soc B Biol Sci. 354(1387):1325-1346. https://doi.org/10.1098/ rstb.1999.0482

$\mathrm{Ng}$ KK et al (2016) Reduced functional segregation between the default mode network and the executive control network in healthy older adults: a longitudinal study. Neuroimage 133:321-330. https://doi. org/10.1016/j.neuroimage.2016.03.029

Nicholls MER et al (2012) The relationship between vertical stimulation and horizontal attentional asymmetries. Q J Exp Psychol (hove) 65(12):2384-2396. https://doi.org/10.1080/17470218. 2012.688979

Nuthmann A, Matthias E (2014) Time course of pseudoneglect in scene viewing. Cortex 52(1):113-119. https://doi.org/10.1016/j.cortex. 2013.11.007
Oldfield RC (1971) The assessment and analysis of handedness: the Edinburgh inventory. Neuropsychologia 9(1):97-113. https://doi. org/10.1016/0028-3932(71)90067-4

Ossandon JP, Onat S, Konig P (2014) Spatial biases in viewing behavior. J vis 14(2):20-20. https://doi.org/10.1167/14.2.20

Paladini RE et al (2017) The influence of alertness on the spatial deployment of visual attention is mediated by the excitability of the posterior parietal cortices. Cereb Cortex 27(1):233-243. https://doi.org/10.1093/cercor/bhw390

Schenkenberg T, Bradford DC, Ajax ET (1980) Line bisection and unilateral visual neglect in patients with neurologic impairment. Neurology 30(5):509-517. https://doi.org/10.1212/wnl.30.5.509

Schmitz R, Peigneux P (2011) Age-related changes in visual pseudoneglect. Brain Cogn 76(3):382-389. https://doi.org/10.1016/j. bandc.2011.04.002

Störmer VS et al (2013) Normal aging delays and compromises early multifocal visual attention during object tracking. J Cogn Neurosci 25(2):188-202. https://doi.org/10.1162/jocn_a_00303

Thomas NA et al (2015) Upper visual field distractors preferentially bias attention to the left. Cortex 64:179-193. https://doi.org/10. 1016/j.cortex.2014.10.018

Urwyler P et al (2015) Age-dependent visual exploration during simulated day- and night driving on a motorway: a cross-sectional study. BMC Geriatr. https://doi.org/10.1186/s12877-015-0015-2

Varnava A, Halligan PW (2007) Influence of age and sex on line bisection: a study of normal performance with implications for visuospatial neglect. Aging Neuropsychol Cogn 14(6):571-585. https:// doi.org/10.1080/13825580600826454

Publisher's Note Springer Nature remains neutral with regard to jurisdictional claims in published maps and institutional affiliations. 